\title{
Die Bilanz der Privatisierung
}

Nach dem Kollaps der realsozialistischen Staaten galt die Eigentumsfrage als historisch gelöst. Privateigentum und Wettbewerb hatten sich als überlegene Form des Wirtschaftens durchgesetzt. Nun ging es auch den Restbeständen staatlichen Unternehmertums an den Kragen: „Privat ist besser" war das Motto der Stunde. Folglich wurde das Tafelsilber - Telekom, Post, Bahn, Energieversorgung - kräftig geputzt und in Teilen verkauft. Der Widerstand gegen den großen Ausverkauf war gering. $\mathrm{Zu}$ schlecht war die Leistungsbilanz der groBen Staatsbetriebe.

Über zwei Jahrzehnte nach den großen Privatisierungs- und Liberalisierungswellen steckt der moderne Kapitalismus in der schwersten Krise seit 80 Jahren. Die globale Wirtschafts- und Finanzkrise hat den Glauben an sich selbst steuernde Märkte erschüttert. Die Enteignung und Kommerzialisierung öffentlichen Eigentums erscheint plötzlich in einem anderen Licht. In der Krise erleben wir eine Renaissance des Staates. Diese historische Krise eröffnet die Chance, das Verhältnis von Staat und Markt neu zu bestimmen.

Hierfür bedarf es auch einer kritischen Bilanz der Privatisierung. Eigentlich wäre dies Aufgabe der Bundesregierung. Aber ein amtlicher Privatisierungsbericht lässt immer noch auf sich warten. Was also hat das privatwirtschaftliche Fitnessprogramm für vermeintlich marode Staatsmonopolisten gebracht?

\section{WENIG LICHT - VIEL SCHATTEN}

Eine effiziente private Unternehmensführung und mehr Wettbewerb sollten sich zuerst auf den Geldbeutel der Verbraucher auswirken. Der durch Monopolpreise und Zwangsgebühren geschröpfte Bürger sollte endlich entlastet werden. Die Vorstellung war, dass die Preise ohne Quersubventionierung, öffentliche Defizitabdeckung, Schonarbeitsplätze, Bürokratie und nicht marktkonforme Löhne bald tieffliegen würden.
Und tatsächlich tanzten die Preise. Telefonieren und Internetsurfen sind billiger geworden. Sparsame Kunden telefonieren heute für einen Cent im Festnetz. Pakete und Päckchen sind hingegen um fast $30 \%$ teurer als zur Jahrtausendwende. Auch das Bahnfahren kostet mehr. In den letzten sieben Jahren stiegen die Preise im Nahverkehr um mehr als ein Fünftel. Bei Strom und Gas bezahlen die Kunden die Extraprofite eines Stromkartells. Die Strompreise für Industriekunden stiegen seit 2000 zwischen $58 \%$ und $77 \%$. Die privaten Haushalte mussten rund $50 \%$ mehr bezahlen. Mehr als die Hälfte dieses Anstiegs geht auf die Preispolitik der Energieversorger zurück. Die Umverteilung bei den Tarifen bzw. Preisen zugunsten der Unternehmen ist dem privatisierungsbedingten Wegfall der Quersubventionierung geschuldet.

$\mathrm{Ob}$ die Preise klettern oder fallen, ist weniger eine Frage des Eigentums als vielmehr der Marktmacht. Während der deutsche Telekommunikationsmarkt der wettbewerbsintensivste in Europa ist, tummeln sich auf dem Energiemarkt ganze vier Konzerne. Diese vier großen Energieversorger stellen heute $82 \%$ der Erzeugungskapazitäten. Sie kontrollieren die gesamte Wertschöpfungskette. Bei Post und Bahn herrscht Nischenwettbewerb. Die konkurrierenden Lizenznehmer der Deutschen Post AG haben nur $9 \%$ des Briefmarktes. Im Regionalverkehr liegt der Marktanteil der DB-Konkurrenz bei $13 \%$. Ungeklärt ist jedoch bis heute, ob sich natürliche Monopole überhaupt in funktionsfähige Wettbewerbsmärkte überführen lassen. Wo dies nicht möglich ist, ist die Rückkehr zu öffentlichem Eigentum die angemessene Antwort.

Dort, wo Preise purzeln, tragen die Beschäftigten die Hauptlast der erfolgreichen Liberalisierung. Bei der Telekom verloren in den letzten zehn Jahren 100.000 Mitarbeiter ihren Job. Die Bahn strich seit der Bahnreform über 150.000 Arbeitsplätze, die Stromwirtschaft etwa 90.000. Bei der
Post kostete die Marktöffnung jeden zweiten heimischen Job. Zwar schufen die Mitbewerber der Staatsriesen neue Arbeitsplätze, der Saldo bleibt jedoch negativ. Die Löhne sinken, da bei Weihnachts- und Urlaubsgeld sowie Zulagen gekürzt wird. Darüber hinaus verschlechtern sich die Arbeitsbedingungen. Da nicht der gesamte Personalabbau rationalisierungsbedingt ist, wird heute länger und härter gearbeitet. Die Abwärtsspirale beschränkt sich nicht auf die Exmonopolisten. Im Gegenteil: Bei der Konkurrenz boomt die prekäre Beschäftigung. Bei den Mitbewerbern der Post zahlen nur zwei von fünf Arbeitnehmern in die Sozialkassen ein. Die Einstiegsgehälter der Konkurrenten liegen bis zu $50 \%$ unter dem Niveau des gelben Riesen. Bei Arcor, Versatel \& Co verdienen die Callcenter-Mitarbeiter jährlich bis zu $17.000 €$ weniger als bei der Telekom.

Aus betriebswirtschaftlicher Sicht bietet das Downsizing (häufig als Gesundschrumpfen verklärt) keinen Anlass zur Sorge. Der Jobabbau stoppt die Subventionierung verdeckter Arbeitslosigkeit. Schlechtere Arbeitsbedingungen beseitigen steuerfinanzierte Privilegien. Aus der gesamtwirtschaftlichen Vogelperspektive sieht die Welt aber ganz anders aus: Personalabbau und Prekarisierungsstrategien verlagern betriebliche Modernisierungskosten auf die Gesellschaft. Die Steuerzahler finanzieren den heutigen realen Kombilohn - Arbeitseinkommen plus Hartz IV - und somit flächendeckendes Lohndumping. Darüber hinaus stehen Beschäftigungsniveau, Löhne und Arbeitsbedingungen in einem engen Verhältnis zur Qualität der Dienstleistungen. Dies gilt besonders für personalintensive Dienste, wie einige Beispiele verdeutlichen: Im Bahnverkehr erhöhen

Dierk Hirschel, Dr., ist Chefökonom beim $D G B$-Bundesvorstand. e-mail: Dierk.Hirschel@bvv.dgb.de 
Personalabbau und Arbeitsverdichtung die Unfallgefahr. Wenn Zustellerbezirke vergrößert werden, erreichen Briefe nur noch verspätet ihre Empfänger. In den Call-Centern sinkt mit Billiglöhnen die Kundenfreundlichkeit. Ein unzureichend qualifiziertes Wartungspersonal gefährdet die Versorgungsqualität der Kraftwerke.

Die Versorgungsqualität ehemals öffentlicher Dienste ist aber auch abhängig von der Investitionsbereitschaft. Kritisch wird es, wenn ein brutaler Preiswettbewerb die Investitionsanreize senkt. Die Infrastrukturen ehemals natürlicher Monopole bedürfen immer wieder großer Modernisierungsinvestitionen. Hinzu kommt, dass erst $\mathrm{ab}$ einer bestimmten Netz- und Betriebsgröße überhaupt Größen- und Verbundvorteile entstehen. Folglich ist der Kapitaleinsatz für Newcomer sehr hoch. Ohne Aussicht auf temporäre Monopolprofite investieren weder die Exmonopolisten noch die Frischlinge. Die volkswirtschaftlichen Kosten unterbliebener Investitionen sind aber höher als die Effizienzverluste aus überhöhten Preisen. Umgekehrt drückt eine zu starke Vermachtung der Märkte ebenfalls auf die Investitionsbremse. Die
Investitionen in Telekommunikation, Elektrizitätswirtschaft und Schienenverkehr sind rückläufig. Der große Stromausfall 2006, die Unterversorgung des ländlichen Raums mit modernen Telekommunikationsdiensten und Verkehrsinfrastruktur sind die Folgen falsch gesetzter ökonomischer Anreize. Das richtige Maß an Wettbewerb wurde hier noch nicht gefunden.

Verschlechtert haben sich auch die $\mathrm{Zu}$ gangsmöglichkeiten einkommensschwacher Bevölkerungsgruppen. Die Post hat inzwischen jeden vierten Briefkasten abgehängt und schließt alle Filialen in Einzugsgebieten unter 2000 Einwohner. Die Bahn strich das beliebte Wochendticket und stellte den InterRegio-Verkehr ein; über 450 Strecken wurden stillgelegt und zahlreiche Bahnhöfe geschlossen. Das neue Glasfasernetz der Telekom schlägt einen Bogen um den ländlichen Raum. Das Universaldienstprinzip bricht sich immer wieder am privaten Gewinnstreben.

\section{FAZIT}

Unter dem Strich überzeugen die Ergebnisse der bisherigen Privatisierung nicht.
Eine billige, flächendeckende und qualitativ hochwertige Versorgung mit öffentlichen Gütern konnte durch die Privatisierungspraxis und Marktöffnung der letzten Jahre nicht erreicht werden - von der Entwicklung der Beschäftigung, der Löhne und Arbeitsbedingungen ganz zu schweigen. Dies muss keine Frage des Eigentums sein. Möglicherweise können die gewünschten Ziele auch durch ein Mehr an Wettbewerb erreicht werden. Vor allem bedarf es aber einer besseren Regulierung: Mindestlöhne, Tariftreueklauseln sowie soziale und ökologische Vergabekriterien können die Gefahr eines reinen Preis- und Kostenwettbewerbs beschränken. Die Überwachung dieser Standards erfordert jedoch ein engmaschiges Regulierungsnetz. Qualität und Umfang der Leistungen müssen vertraglich festgelegt werden. Dadurch entstehen hohe Transaktionskosten. Ungleiche Machtverhältnisse erleichtern es Konzernen, die Vertragsbedingungen zu diktieren. Zudem ist Regulierung immer auch korruptionsanfällig. Wenn der Regulierungsaufwand aber ins Unendliche steigt und die gewünschten Ergebnisse ausbleiben, dann stellt sich die Eigentumsfrage neu. 